health laboratories, modify surveillance systems for enhanced data collection and rapid communication of AST results, and initiate partner services and investigations among patients with GC demonstrating reduced susceptibility (RS) to ceftriaxone, cefixime or azithromycin.

Outcomes/Impact SURRG grantees incorporated robust genital, pharyngeal, and rectal gonococcal culture collection from all genders at participating clinics. During 2018-2019, grantees performed AST on $>10,700$ isolates with a five-day median turnaround time from specimen collection to reporting AST results to providers. Fifty-nine percent of patients with RS GC returned for a test-of-cure; no resistance-related treatment failures were detected. Among 4,511 isolates, we found $\geq 95 \%$ concordance (within one doubling dilution) between AST performed locally using Etest ${ }^{\circledR}$ compared to agar dilution (reference method) for ceftriaxone, cefixime and azithromycin. We conducted investigations among cases and partners, identifying $>100$ new GC cases. Finally, we merged epidemiologic and partner data with isolate genomic data to further explore sexual networks with GC transmission and identify opportunities for local interventions.

Innovation and Significance SURRG successfully built clinic, laboratory, and epidemiological capacity for local ARGC rapid detection and response. Notable outcomes/innovations include establishing best practices for collecting and transporting gonococcal culture specimens, implementing Etest ${ }^{\circledR}$ in local jurisdictions, and measuring the value of containing ARGC spread through partner services. Lessons learned and project-informed identification of additional ARGC control needs at the local and national level are being used to inform CDC's ongoing ARGC control efforts.

\section{O08.5 IMPACT OF INCREASED ASYMPTOMATIC TESTING FOR GONORRHOEA AMONG MSM ON PREP ON THE TRANSMISSION AND DEVELOPMENT OF ANTIMICROBIAL RESISTANCE}

1J Heijne*, ${ }^{1} \mathrm{M}$ Visser, ${ }^{2} \mathrm{~J}$ Riou, ${ }^{2} \mathrm{~N}$ Low. ${ }^{1}$ National Institute for Public Health and the Environment (RIVM), Bilthoven, The Netherlands; ${ }^{2}$ University of Bern, Bern, Switzerland

\subsection{6/sextrans-2021-sti.96}

Background In many countries, men who have sex with men (MSM) using pre-exposure prophylaxis (PrEP) are routinely three-monthly tested and treated for Neisseria gonorrhoeae (gonorrhoea), which might increase antimicrobial resistance (AMR). However, there is uncertainty about how AMR emerges. We investigated the impact of frequent testing and treating on gonorrhoea prevalence and AMR assuming different ways of AMR emergence.

Methods We developed a compartmental model, which included three sexual activity classes that differed in unprotected sex partner numbers and testing uptake. AMR to ceftriaxone was incorporated as stepwise increases in minimum inhibitory concentrations and eventual resistance (MIC drift) in three separate ways: direct selection pressure through ceftriaxone treatment; indirect selection pressure through antimicrobials treatment for other reasons; or imported infection. Each model was calibrated to the ceftriaxone MIC curve from the Dutch Gonococcal Resistance to Antimicrobials Surveillance program (2015-2019). We estimated the impact of three-monthly testing of high-risk MSM on overall and AMR prevalence in 2030 .
Results Overall gonorrhoea model prevalence was 3.4\% (95\% uncertainty interval $3.2 \%-3.5 \%)$, and $14.3 \%(11.7 \%-18.0 \%)$ in high-risk MSM. Three-monthly testing resulted in 66\%93\% reductions in overall prevalence for all three ways in which AMR development was modelled. AMR prevalence was low in 2030 for all three mechanisms, but increased fastest with direct selection compared to indirect or imported. With direct selection, AMR prevalence was 1.7 (1.3-2.0) times higher than with indirect selection and 8.2 (2.7-16.1) times higher than through imported infection. When high-risk MSM increased numbers of unprotected sex partners, three-monthly testing reduced overall prevalence less and AMR prevalence increased, especially with direct selection.

Conclusion With frequent testing of MSM, direct selection pressure through treatment leads to more gonococcal resistance than indirect selection or imported infection. This study highlights the need to increase our understanding of mechanisms of AMR emergence in $\mathrm{N}$. gonorrhoeae.

\subsection{ASSESSING WITHIN-HOST GENETIC VARIATION IN NEISSERIA GONORRHOEAE AT DIFFERENT ANATOMICAL LOCATIONS AND OVER TIME}

1,2J de Korne* ${ }^{*}$ 1,3S Bruisten, ${ }^{4,5} \mathrm{H}$ de Vries, ${ }^{1,2} \mathrm{~A}$ van Dam. ${ }^{1}$ Department of Infectious Diseases, Public Health Laboratory, Public Health Service of Amsterdam, Amsterdam, The Netherlands; ${ }^{2}$ Amsterdam UMC, University of Amsterdam, Department of Medical Microbiology, Amsterdam Institute for Infection and Immunity (All), location Academic Medical Center, Amsterdam, The Netherlands; ${ }^{3}$ Amsterdam UMC, University of Amsterdam, Amsterdam Institute for Infection and Immunity (AlI), location Academic Medical Center, Amsterdam, The Netherlands; ${ }^{4}$ Amsterdam UMC, University of Amsterdam, Department of Dermatology, Amsterdam Institute for Infection and Immunity (All), location Academic Medical Center, Amsterdam, The Netherlands; ${ }^{5}$ STI Outpatient Clinic, Department of Infectious Diseases, Public Health Service Amsterdam, Amsterdam, The Netherlands

\subsection{6/sextrans-2021-sti.97}

Background Recent trials focus on novel antibiotic treatment options for Neisseria gonorrhoeae $(\mathrm{Ng})$, since resistance against currently used cephalosporins is emerging. To assess antibiotic potential, patients with mixed infections or those who are reinfected after treatment should be identified. Comparing levels of within-host genetic variation in $\mathrm{Ng}$ isolates at different anatomical locations and over time could identify those patients and could inform thresholds for similarity.

Methods Isolates from the New AntiBiotic treatment Options for uncomplicated GOnorrhoea (NABOGO) trial were used in this study. Whole-genome sequences were obtained from paired isolates from 86 patients with multiple isolates from different anatomical locations (n pairs $=59$ ) or from different time points ( $\mathrm{n}$ pairs $=48$ ). All isolates were typed according to the typing schemes: NG-Multi-Antigen Sequence Typing (NGMAST), NG-Sequence Typing for Antimicrobial Resistance (NG-STAR), Multi-Locus Sequence Typing (MLST) and core genome MLST (cgMLST).

Results Identical NG-MAST/NG-STAR/MLST profiles were identified within 93/107 (87\%) isolate pairs. Different strains were found in $8 / 59(14 \%)$ paired isolates from different anatomical locations, according to completely different NG-MAST/NG-STAR/MLST profiles. Five other isolate pairs from multiple anatomical locations differed in NG-MAST but were similar in NG-STAR/MLST. One case of reinfection was identified $(1 / 48,2 \%)$ according to 